clinical trial were provided by AbbVie. AbbVie participated in the interpretation of data, drafting, review, and approval of the abstract.

Disclosure of Interest: D. Opris-Belinski Consultant for: Abbvie, BMS, Pfizer, Roche, Teva and consulting fees from Abbvie, BMS, Pfizer, Roche, Teva., S. Erdes Consultant for: Abbvie, MSD, Pfizer, USB, BIOCARD, Gedeon Richter, Dr. Reddy's, Novartis., S. Grazio Consultant for: Abbvie/Abbott Lab, Roche, MSD, Eli Lilly, Pfizer, Boehringer Ingelheim, Grünenthal, Stada, Sanofi-Aventis, PharmaSwiss, Berlin-Chemie, Pliva/Teva, Belupo, Krka, consulting fees from: Abbvie/Abbott Lab, Roche, MSD, Eli Lilly, Pfizer, Grünenthal, L. Šenolt Consultant for: AbbVie, Bristol-Myers Squibb, Celgene Corporation, Eli Lilly, Merck Sharp and Dohme, Novartis, Pfizer, Samsung, Takeda, UCB., M. Hojnik Employee of: AbbVie, O. Nagy Employee of: AbbVie, L. losub Employee of: AbbVie, S. Szántó Consultant for: Abbvie, Bristol Myers-Squibb, Novartis, Pfizer, Roche, Teva DOI: 10.1136/annrheumdis-2017-eular.5244

\section{THU0364 IMMUNOGENICITY OF ANTI-TNF DRUGS AND CLINICAL RESPONSE IN PATIENTS WITH SPONDYLOARTHRITIS}

D. Geiger ${ }^{1}$, Y. Degboe ${ }^{1}$, T. Barnetche ${ }^{2}$, A. Cantagrel ${ }^{1}$, A. Ruyssen-Witrand ${ }^{1}$, A. Constantin ${ }^{1} .{ }^{1}$ Rheumatology, Centre de Rhumatologie, CHU Purpan, Place du Dr Baylac, Toulouse Cedex $9 ;{ }^{2}$ Rheumatology, Centre Hospitalier Universitaire de Bordeaux, Bordeaux, France

Background: Antidrug antibodies (ADAb) seem to be associated with a loss of response in immune-mediated inflammatory diseases (1) and in psoriatic arthritis (2). Objectives: To assess the effect of ADAb on clinical response in patients with spondyloarthritis $(\mathrm{SpA})$ treated with anti-TNF drugs.

Methods: We conducted a systematic literature review of controlled trials and observational studies assessing the effect of ADAb on response to anti-TNF drugs (Adalimumab (ADL), Certolizumab (CTZ), Etanercept (ETA), Golimumab (GOL) and Infliximab (INF)) in patients with axial or peripheral SpA. Databases analysed were PubMed, the Cochrane library, and ACR/EULAR meeting abstracts, until January 2017. A meta-analysis was performed using the inverse variance approach and statistical heterogeneity was assessed with the Cochran Q-test and $\mathrm{I}^{2}$ values. A statistical threshold of $5 \%$ was considered as significant.

Results: Over 1,387 publications screened, 7 studies were selected for metaanalysis (3-9). These studies were observational studies $(n=6)$ or controlled trial $(n=1)$; involved patients with axial or peripheral $\operatorname{SpA}(n=6)$ or psoriatic arthritis $(n=1)$; included treatments with ADL $(n=4)$, ETA $(n=1)$, INF and INF biosimilar $(n=2)$, or various anti-TNF drugs $(n=1)$. ADAb rates varied between anti-TNF drugs: $0 \%$ for ETA, $13.6-31.4 \%$ for ADL, $0-28.9 \%$ for INF. Patients with ADAb were less often responders than patients without ADAb in 6 studies, more often responders in one study, while the risk ratio (RR) for response was not assessable in one study due to the absence of ADAb. The weighted pooled RR $(95 \% \mathrm{Cl})$ for response to anti-TNF drugs was $0.73(0.54-0.98)$ in ADAb+ in comparison with ADAb- patients $(p=0.04)$ (see figure). There were trends towards more infusion reactions and lower serum drug levels in patients with ADAb (data not shown).

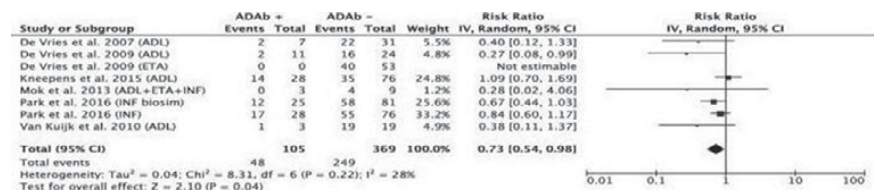

Conclusions: According to the results of this meta-analysis, ADAb positivity is associated with a lower rate of response to anti-TNF agents in patients with SpA. References:

[1] The immunogenicity of anti-TNF therapy in immune-mediated inflammatory diseases: a systematic review of the literature with a meta-analysis, Garcês.S and al, ARD 2014; 72:1947-1955.

[2] The comparative immunogenicity of biologic therapy and it's clinical releavance in psoriatic arthritis: a systematic review of the literature, Balsa.A and al, ACR 2016, Abstract number 1691.

Disclosure of Interest: None declared

DOI: 10.1136/annrheumdis-2017-eular.5726

\section{THU0365 DO EXTRA-ARTICULAR MANIFESTATIONS AFFECT THE CHOICE OF BIOLOGIC THERAPY IN PATIENTS WITH AXIAL SPONDYLOARTHRITIS? A MULTICENTRE REAL-LIFE ANALYSIS}

E.G. Favalli ${ }^{1}$, S. D'Angelo ${ }^{2}$, A. Carletto ${ }^{3}$, A. Becciolini ${ }^{1}$, F. Martinis ${ }^{3}$, G. Tramontano ${ }^{2}$, M.G. Raimondo ${ }^{4}$, M. Biggioggero ${ }^{4}$, A. Marchesoni ${ }^{1}$ M. Rossini ${ }^{3}$, I. Olivieri ${ }^{2} .{ }^{1}$ Department of Rheumatology, Gaetano Pini Institute, Milano; ${ }^{2}$ Rheumatology Institute of Lucania (IRel) - Rheumatology Department of Lucania, San Carlo Hospital of Potenza and Madonna delle Grazie Hospital of Matera, Potenza; ${ }^{3}$ UOC Reumatologia, Dipartimento di Medicina, AOUI, Verona; ${ }^{4}$ Department of Clinical Sciences and Community Health, Division of Rheumatology, University of Milan and Gaetano Pini Institute, Milano, Italy

Background: Extra-articular manifestations (EAMs), such as uveitis, inflammatory bowel diseases (IBD) and psoriasis (PsO), frequently complicate the disease course of patients with axial spondyloarthritis $(\mathrm{axSpA})$, although prevalence data on this regard are still controversial. The occurrence of EAMs might also contribute to the decision of introducing a biologic therapy and even influence the choice between the available TNF inhibitors (TNFis).

Objectives: The aim of this study is to retrospectively evaluate the prevalence of EAMs in a multicentre cohort of axSpA patients treated with TNFi, investigating how these influenced the choice of treatment.

Methods: Clinical data from axSpA patients treated with a TNFi between May 2003 and May 2016 where obtained from a multicentre registry. Prevalence of EAMs (uveitis, IBD and PsO) was calculated at the time of TNFi prescription, evaluating their distribution according to drug subgroup.

Results: The study included 503 patients with axSpA (172 [34.2\%] women, mean age $[ \pm S D] 40.5[ \pm 13.2]$ years, mean disease duration $9.7[ \pm 14.7]$ years), receiving a total of 675 lines of treatment (I-line $n=503$, II-line $n=118, \geq$ III-line $n=54$ ) with a TNFi (272 infliximab [IFX], 173 adalimumab [ADA], 89 golimumab [GOL], 141 etanercept [ETN]). At the time of TNFi introduction, $28.6 \%$ patients claimed at least one EAM (IBD 11.3\%, uveitis 10.9\%, and PsO 8.8\%). The baseline presence of at least one EAM was associated with a more frequent prescription of an anti-TNF monoclonal antibody rather than etanercept (34.1\% versus $21.9 \%$, respectively; $p=0.005$ ). In detail, EAMs were found in $41.6,36.9,29.8$, and $21.9 \%$ patients treated with GOL, ADA, IFX, or ETN, respectively. The prevalence of IBD was significantly higher $(\mathrm{p}=0.004)$ in patients treated with ADA $(12.7 \%)$, IFX $(14.3 \%)$, or GOL $(11.2 \%)$ compared with ETN $(4.9 \%)$. Uveitis was numerically more frequent in GOL $(20.2 \%)$ and ADA (13.3\%) rather than IFX (9.5\%) and ETN $(9.9 \%)$ groups. Finally, PsO prevalence was similar in patients treated with ADA $(10.9 \%)$ and GOL $(10.1 \%)$, and numerically lower in the ETN $(7.1 \%)$ and IFX (5.9\%) groups.

Conclusions: In our cohort of axSpA patients treated with TNFis, EAMs were highly represented. The presence of extra-articular involvement has been carefully taken into account when a TNFi was required to better control the disease. In particular, IBD and uveitis drove more frequently the choice toward an anti-TNF monoclonal antibody instead of the receptor.

Disclosure of Interest: None declared

DOI: 10.1136/annrheumdis-2017-eular.3779

\section{THU0366 GASTROINTESTINAL INFECTIONS IN PATIENTS WITH SPONDYLOARTHRITIS TREATED WITH ANTI-TNF DRUGS: RESULTS OF GISEA REGISTER}

F. Atzeni ${ }^{1}$, V. Panetta ${ }^{2}$, M. Sebastiani ${ }^{3}$, F. Salaffi ${ }^{4}$, A. Carletto ${ }^{5}$, R. Foti ${ }^{6}$, F. lannone $^{7}$, G. Elisa ${ }^{8}$, M. Govoni $^{9}$, A. Marchesoni ${ }^{10}$, E.G. Favalli ${ }^{10}$, R. Gorla ${ }^{11}$, R. Ramonda ${ }^{12}$, P. Sarzi-Puttini ${ }^{13}, G$. Ferraccioli ${ }^{8}, G$. Lapadula ${ }^{7}$ on behalf of GISEA group. ${ }^{1}$ Rheumatology Unit, University Hospital L. Sacco, Milan, Italy, Milan; ' 2 'altrastatistica Consultancy \& Training, Biostatistics Office, Rome; ${ }^{3}$ University Hospital of Modena, Modena; ${ }^{4}$ Polytechnic University of Marche, C. Urbani Hospital, Jesi; ${ }^{5}$ Rheumatology Unit, University of Verona, Verona; ${ }^{6}$ Rheumatology Unit, Vittorio-Emanuele University Hospital of Catania, Catania; ${ }^{7}$ University of Bari, Bari; ${ }^{8}$ Division of Rheumatology, Institute of Rheumatology, Catholic University of the Sacred Heart, Rome; ${ }^{9}$ Department of Medical Sciences, UOC of Rheumatology, Santa Anna University Hospital, Ferrara; ${ }^{10} \mathrm{G}$. Pini Orthopedic Institute, Milan, Milan; ${ }^{11}$ Rheumatology and Immunology Unit, Spedali Civili, Brescia; ${ }^{12}$ University of Padua, Padua; ${ }^{13}$ Rheumatology Unit, University Hospital L.Sacco, Milan, Italy

Background: Tumour necrosis factor (TNF) plays a pivotal role in controlling intracellular of bacterial infection. The BSR Biologics Register (BSRBR) has reported an increase in the occurrence of listeria and salmonella infections in anti-TNF-treated rheumatoid arthritis patients in comparison with those patients treated with non-biological DMARDs.

Objectives: The aim of this study was to determine the incidence of gastrointestinal infection in the anti-TNF-treated spondyloarthritis (SpA) patients in the GISEA registry, and identify the factors associated with its development.

Methods: The prospective GISEA registry was designed to collect real-world clinical data concerning patients with RA or $\mathrm{SpA}$ treated with biological drugs. The baseline information includes demographics, disease duration, HAQ-DI, DAS-28, BASDAI, BASFI and BASMI scores, steroid use, smoking history and comorbidities.

Results: Of the 3321 anti-TNF-treated SpA patients in the registry (1731 males, $52.2 \%$; mean age $47 \pm 13$ years; median disease duration three years, interquartile range [IQR] 0-8), 1065 (32\%) were treated with infliximab (IFN), 1052 (32\%) with adalimumab (ADA), and $1204(36 \%)$ with etanercept (ETN). Two thousand, one hundred and five patients $(63.4 \%$ ) had a median of one comorbidity (IQR 0-2], the most frequent being hypertension (701), thyroid diseases (281), diabetes mellitus (207), cardiopathy (189), and osteoporosis (145). In combination with the biological drug, 919 patients $(27.7 \%)$ received steroids and $2451(79.9 \%)$ at least one DMARD. The median follow-up was three months (IQR 1-2 years). Twenty-two patients $0.7 \%$ experienced bacterial gastrointestinal infections, the most frequent being due to listeria, klebsilla and salmonella. The crude incidence rate was 2.5 per 1000 patient-years $(95 \% \mathrm{Cl} 1.6-3.7)$. Univariate analysis showed that female gender (OR 3.9, 95\% Cl 1.5-10.0; $\mathrm{p}=0.004$ ) and comorbidities (OR $3.4,95 \% \mathrm{Cl} 1.0-3.5 ; \mathrm{p}=0.049$ ) were associated with a high risk of gastrointestinal infections, and that the use of IFN rather than ETN and ADA ( $p=0.712$ and $p=0.238$ ) was not associated with a higher risk of gastrointestinal infections. 